\title{
Using Word Embedding to Reveal Monetary Policy Explanation Changes
}

\author{
Akira Matsui, Xiang Ren, Emilio Ferrara \\ University of Southern California \\ \{amatsui, xiangren, emiliofe\}@usc.edu
}

\begin{abstract}
Documents have been an essential tool of communication for governments to announce their policy operations. Most policy announcements have taken the form of text to inform their new policies or changes to the public. To understand such policymakers' communication, many researchers exploit published policy documents. However, the methods wellused in other research domains such as sentiment analysis or topic modeling are not suitable for studying policy communications. Their training corpora and methods are not for policy documents where technical terminologies are used, and sentiment expressions are refrained. We leverage word embedding techniques to extract semantic changes in the monetary policy documents. Our empirical study shows that the policymaker uses different semantics according to the type of documents when they change their policy.
\end{abstract}

\section{Introduction}

Disentangling how policymakers explain their policy is crucial to understand how policymakers supported by democracy uphold their responsibility. However, extracting such abstract information from policy documents is challenging. First of all, policy documents are often crabbedly written with technical terminologies, meaning that machine learning models trained on general corpora may not work well. In addition, popular text mining techniques for social science such as sentiment analysis or topic modeling may not be applicable. It would be a strong assumption, for example, that changes in policy explanation reflect its sentiments. Even if we observed topic changes in documents during policy changes, it only tells "what" was explained but does not disclose "how" policy explanations changed.

This paper proposes a word embedding-based framework for understanding how policymakers change their explanation when they change their

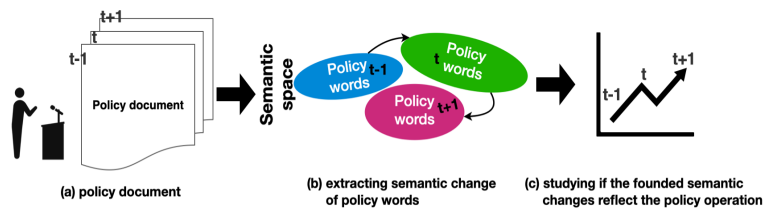

Figure 1: Schematic of our framework and study design

policies. Our framework trains the word embedding model only on policy documents, which allows us to resolve the aforementioned challenges by defining the semantic of the policy words solely on the policy documents. We use our approach to study the monetary policy operation by the Federal Reserve Bank (FRB) in the united states. Our framework reveals how the policymaker explains their policy change in the policy documents and finds that different policy documents have a different role in policy change explanation even though the same policymaker publishes these documents. We also find that our word embedding-based framework can be used to predict policy changes. We summarize our framework and study design at Figure 1.

\section{Methods and Data}

\subsection{Word embedding}

To extract the policy explanation from the policy documents, we use a dynamic embedding model, focusing on the transition of the semantic of the important words in the policy. The dynamic embedding calculates the semantic differences of the words in the documents over time. Hence, it allows us to study the differences in the semantics of specific policy-related words between two consecutive periods. Word embeddings highlight the semantic movements hidden in corpora, such as gender biases (Garg et al., 2018) or law-of semantics (Hamilton et al., 2016). Comparing the embedding vectors from the corpora over time can yield fallacious analysis because low-dimension embed- 
ding vectors can yield arbitrary orthogonal transformations (Hamilton et al., 2016). To avoid this pitfall, we use the state-of-the-art dynamic wordembedding method that efficiently yields temporal word embeddings (Di Carlo et al., 2019). We construct word-embedding vectors for each time, $\boldsymbol{w}_{\boldsymbol{t}}$ (30 dimensions).

\subsubsection{Introducing the reference words}

To obtain interpretable measurement of policy explanation change, we quantify the semantic distance between the policy-related and the reference words. While we can calculate the cosine similarity between the word embedding vectors of the words of interest as (Hamilton et al., 2016), these similarities only describe the degree of semantic changes but not "how" the semantics of policy words change. Instead, we capture the semantic changes of the policy words by calculating how the semantic of policy words are close to the reference words as (Garg et al., 2018).

For the reference words, we use the set of neutral words and the set of positive and negative words from the Subjectivity Lexicon (Wilson, 2005; Wilson et al., 2005). The Subjectivity Lexicon is the annotated polarity dictionary of subjective words: words with explicit polarity (positive and negative), and neutral words ${ }^{1}$.

We use these annotated words to calculate the relative distance difference between the semantic of policy words and explicit semantic (positive and negative) words or neutral semantic (neutral) words. We also try another setting of the reference words calculating the relative distance difference between the semantic of policy words and positive semantic words or negative semantic words.

The role of these reference words in this paper is not to measure polarity but rather to measure the degree of the policy-related explanations become explicit during policy changes. To do so, we measure the semantic distance between explicit reference words and policy-related words (the detail to be discussed in Eq 1 in the next subsection). Also, since the embedding vectors of the reference words will be also learned from the policy document, the semantic of such reference words also reflect the text specificity of the policy. With this advantage, our framework overcomes the problem of language

\footnotetext{
${ }^{1}$ For example, the Subjectivity Lexicon annotates "clear", "vague" and "completely" as positive, negative, neutral subjective words respectively. The Subjectivity Lexicon is publicly available at (Wilson, 2005).
}

model trained on general corpora discussed in the introduction.

\subsubsection{Calculating relative distances of the reference words to the policy words}

With the trained word embedding model on policy documents, we will measure how the policymaker changes their policy explanation over policy operations. In this study, we calculate the degree of explicit of the policy words that measure the semantic distance between the important policy words and the reference words that have particular stable semantics.

To represent the degree of explicit of policy explanation, we calculate the relative distance between the reference words and the policy words. To easy to follow our analysis, we summarize the selection of reference words in Table 1. Following (Garg et al., 2018), we calculate the semantic differences at each time period $t$ as

$$
\begin{aligned}
& R T\left(R e f_{1}, R e f_{2}\right)= \\
& \frac{1}{\|\boldsymbol{E}\|} \sum_{\boldsymbol{w}_{t}^{e} \in \boldsymbol{E}}\left\|\boldsymbol{w}_{t}^{e}-\overline{\boldsymbol{w}}_{t}^{R e f 1}\right\|_{2}-\left\|\boldsymbol{w}_{t}^{e}-\overline{\boldsymbol{w}}_{t}^{\operatorname{Ref} 2}\right\|_{2},
\end{aligned}
$$

where $\boldsymbol{w}_{t}^{e}$ is the semantic vectors of the policy words $p$, at time $t ; \overline{\boldsymbol{w}}_{t}^{\text {Refi }}$ is the average word embedding vectors of the group $i$, where $R e f_{i}$ are the set of subjective words; $\|\boldsymbol{E}\|$ is the number of policy words studied.

Eq 1 calculates the relative similarity of the policy words to the set of words $R e f_{1}$ against $R e f_{2}$. When $R e f_{1}$ is a neutral word and $R e f_{2}$ is the concatenation of positive and negative words, a small value of $R T\left(R e f_{1}, R e f_{2}\right)$ indicates that the policy words are close to the explicit semantic lather than the neutral semantic. We call this variable the degree of explicit. For comparison, we also try to set $R e f_{2}$ positive be and $R e f_{1}$ be negative, and it takes a small value when the policy words are close to the positive subjective semantic. We call this variable the degree of positive. We will discuss the selection of the policy words in Sec 2.3.

\subsection{Vector autoregression model (VAR)}

We study whether the calculated degree of explicit policy explanation reflects the actual policy changes over the course of policy operation. The variables of interest, however, have simultaneous interactions with each other. While a semantic shift in policy explanation can follow a change in policy operation, this relation can be the other way around. 
Table 1: The subjective word choice for Relative distance metrics for policy explanation

\begin{tabular}{lll}
\hline Name & $R e f_{1}$ & $R e f_{2}$ \\
\hline 1 & $\begin{array}{l}\text { the degree of explicit } \\
\text { the degree of positive }\end{array} \quad \begin{array}{l}\text { Explicit semantic words (positive and negative words) } \\
\text { Negative semantic words }\end{array}$ & $\begin{array}{l}\text { Neutral semantic words (neutral words) } \\
\text { Positive semantic words }\end{array}$ \\
\hline Note: & The selection of the reference word used in Equation 1. We calculate the relative distance of the word embedding \\
vectors of the policy words and the explicit semantic words, the neutral semantic words (the degree of explicit). For \\
an alternative, we also calculate the degree of positive using the relative distance between the policy word and Negative \\
semantic and Positive semantic (the degree of positive).
\end{tabular}

Taking into account this typical challenge in time series analysis, we employ the vector autoregression model (VAR). VAR is a time series model well used in the application of time series analysis in the financial econometric model (Stock and Watson, 2001; Stock et al., 2003).

VAR is a generalized autoregression model widely used for economic time series analysis with multiple variables. An advantage of the VAR model is that the VAR studies the interactions among variables across time periods. We estimate the VAR model that has the following autoregressive structure,

$$
y_{t}=c+\Phi_{1} y_{t-1}+\cdots+\Phi_{p} y_{t-p}+\epsilon_{t}
$$

where $c$ is $n \times 1$ interception vector; $\Phi$ is $n \times n$ coefficient matrix; $\epsilon_{t}$ is a error term, $\epsilon_{\mathbf{t}} \sim W . N .(\boldsymbol{\Sigma})$ and $\boldsymbol{\Sigma}$ is variance-covariance matrix. Note that $\boldsymbol{y}_{\boldsymbol{t}}$ is the vector that has the variables of log difference at time $t . y_{t}$ such as the degree of explicit, the representation of policy operation discussed in the following section.

We use the estimated VAR model to study if a change in the semantic of the policy explanation can follow the policy change. To do so, we conduct impulse response simulation, which is one of the standard methods in financial econometric analysis (Stock and Watson, 2001; Stock et al., 2003). Impulse response simulation investigates how a change in a variable at time $t$ transmits the other variables in the terms after time $t$, modeling the interactions among variables. In our analysis, we study if a change in the variable of the policy semantic at time $t$ transmit the variables represents the policy change at time $t+1$ and after $^{2}$. By doing this, we can remove the relation that the policy change at time $t$ leads to some changes in the semantic of the policy explanation $t$, which is known as simultaneous interactions.

\footnotetext{
${ }^{2}$ (Stock and Watson, 2001) provides a good summary of how to interprets the impulse response analysis with an example in p106.
}

\subsection{Policy words selection and FRB data}

This paper will use the data from FRB. The FRB policy documents explain their policy operation as the Federal law requires. Also, it is widely recognized that maintaining transparency is a key to conduct a successful monetary policy operation theoretically and practically (Woodford, 2005; Geraats, 2002; Blinder et al., 2008). In addition, the FRB publishes the statement about their policy operation, which enables us to select what policy words should be studied in our analysis. Lastly, we measure the FRB policy operation by its policy interest rate. These practical and technical advantages allow us to investigate our research question with our framework.

To construct the variable of the policy words' semantics, using Equation 1, we select the specific groups of words that represent the FRB's monetary policy. For selecting such words, we leverage the situation that the FRB discloses their policy goal as the Fed's mandate (the Federal Reserve Bank of Chicago, 2020). The Fed's mandate states the three main goals of the monetary policy declared by the FRB: "full employment", "stable prices", and "moderate long-term interest rates" (Bank, 2020c; the Federal Reserve Bank of Chicago, 2020). We select the words related to the Fed's mandate: Employment (unemployment rate, wage); Stable prices (unemployment rate, wage, unemployment, labor, full employment); Interest rates (interest, interest rate). As a sub-analysis, we expand the words to study by adding the other terminologies in economics using a economic dictionary by (Welker, 2020).

We construct our corpus of the FRB documents (Bank, 2020a,b): Beige Book, Tealbook, and Federal Open Market Committee (FOMC) minute. These documents inherently contain information on FRB's policy-making, yet they describe the FRB monetary policy from different perspectives. The FOMC minutes is one of the most relevant docu- 
ments that communicates the FRB monetary policy operation. FOMC minute is the summary of the FOMC meetings where the FRB committees decide the direction of the FRB monetary policy. The FOMC committee refers to the Tealbook that reports the economic conditions and backgrounds for monetary policy operation. The Beige Book summarizes the US economic conditions reported by each district of the FRB. The text sizes of each document per quarter are as follow; Beige book (mean:13,952 total: 1,018,531); Minute (mean: 11,075 total: 808,501); Tealbook (mean:68,50 total: $5,000,916)$.

To study whether our framework captures the FRB policy, we need to have a variable that represents the FRB policy. We use the FFR, the interest rate for depository institutions trading federal funds with each other overnight. In this study, we focus on the relationship between the constructed feature and FFR.

\section{Results}

This section reports the results of the impulse response analysis, and the prediction analysis.

\subsection{VAR analysis}

We estimate the VAR model with the degree of explicit in policy documents, FFR and U.S. GDP. We present our impulse simulation with the estimated VAR model at Figure 2. Each sub-figure demonstrates how one-standard deviation positive changes in the degree of explicit transmits the policy operation represented by the federal funds rate (FFR). The blue line represents the percent changes in the policy rate derived from a change in the estimated model.

The first left sub-figure at Figure 2(b) shows that when the policymaker increases the degree of explicit in the document (Tealbook), they increase their policy rate in the next few quarters (the dotted line represent $95 \%$ confidence intervals). On the other hand, the right sub-figure at Figure 2(b) demonstrates that when the policymaker increases the degree of explicit, they will decrease the policy rate in the following few quarters. These results imply that even when simultaneously considered, we found the policymaker changes the semantic of the document when they change their policy.

Our results also reveal that the policy documents have different role in explaining policy changes. Minute and Tealbook show opposite di-
Table 2: Comparing the prediction performances

\begin{tabular}{lccc}
\hline & & Economic Dictionary & Fed's mandate \\
\hline VAR & Deg of explicit & 0.033979 & $\mathbf{0 . 0 3 3 0 0 0}$ \\
& Deg of positive & 0.035895 & 0.035793 \\
& Cosine dist & 0.034892 & 0.035440 \\
& LSA & 0.035426 & \\
& Word count & 0.036309 & 0.037934 \\
\hline LSTM & Deg of explicit & 0.043946 & 0.043875 \\
& Deg of positive & 0.043871 & 0.043743 \\
& Cosine dist & 0.043799 & 0.043633 \\
& LSA & 0.043682 & 0.043692 \\
\hline ARIMA & Word count & \multicolumn{2}{c}{0.037722} \\
\hline
\end{tabular}

Predicting the policy behavior represented by the Federal Funds Rate (FFR). RMSE of the prediction results (40-time series splits data; smaller is better). Deg of explicit and positive are the degree of explicit and positive respectively (summarized at Table 1). Cosine distance calculates the distance average embedding of the policy words between periods. LSA is used to calculate cosine distance of the document vector by Latent Semantic Analysis (LSA) using Bag of Words. Word count is the number of policy words for each reference words.

rection changes. The results also demonstrate that the model with focused reference words (Fed's Mandate) show relatively clearer result than one with the expanded reference words (Economic Dictionary). The left sub-figure of Figure 2(a) does not shows significant policy changes compared to the left sub-figure of Figure 2(b). This result imply that the focused policy related words well captures the policy operation than the general terminologies.

\subsubsection{Implication of the VAR analysis}

The results in Figure 2(b) suggest that the policymaker use the different documents according to the phase of policy. The policymaker increases the degree of explicit in Tealbook when they increases the policy rate (tamping down stimulating economy), and they increases the degree of explicit in the minute when they decrease the policy rate (stimulating economy). Those results clearly describe how the policymaker uses the different documents according to the phase of the policy. When they can write the condition of the economy (in the Tealbook) explicitly, the policymaker tamps down their policy. On the other hand, when the committee's discussion of the policy operation (in the minute) is explicit, they stimulate the economy. To summarize those results, the policy implication of the results is that the policymaker uses the economic condition to rational for tamping down their policy, whereas they stimulate the economy when the committee has a explicit discussion in the policy operation meeting (FOMC meeting). 




\section{Economic Dictionary}
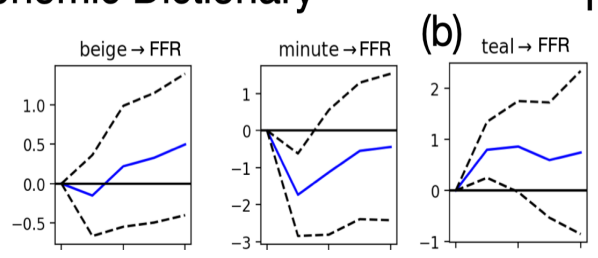

Fed's Mandate

Figure 2: Impulse response analysis

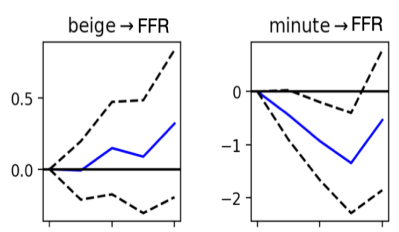

Impulse response to a shock in the neutrality of the policy words in the three documents (FFR: Federal Funds Rate;teal: Tealbook; beige: Beige book; minute: FOMC minute). The $\mathrm{x}$-axis represents the time after a change in the degree of explicit occur (quarterly). The y-axis represents the percent change. The dashed lines represent $95 \%$ confidence interval. They demonstrate how a change in document transmits to the FFR across the following four quarters. Each column represents policy word selection (the economic dictionary, the Fed's mandate)

\subsection{Prediction analysis}

We reports the prediction results in Table 2. For semantic calculation, we use two document-level features: the embedding results of Latent Semantic Analysis and the count of the reference words. We study the cosine distance of the embedding vector of each set of reference words. For prediction models, in addition to VAR, we use LSTM and ARIMA models as a baseline. The performances are measured by the root mean square error (RMSE) between forecasts by the models and the actual outcome. To prevent data leakage, we split the data based on time series split.

Table 2 shows that the degree of explicit returns the better performance than the economic dictionary in most cases, which is consistent with the impulse response simulation. We also find that the predictions by the LSTM model do not return clear results and yield relatively inferior results. We believe this is because of the small data size, as discussed in (Makridakis et al., 2018). This is consistent with the fact that the ARIMA model outperforms the LSTM model even when the ARIMA model uses only the FFR. The result also shows that the performance with Fed's mandate is better than we expand the expanded reference words with more general words about economics from the economic dictionary (Welker, 2020).

\section{Discussion and Conclusion}

This paper has presented the framework to study the policy explanation using the published policy documents. We utilized the word embedding technique to extract the semantic changes of policyrelated words. Our framework provided the interpretable analysis, calculating the semantics between the policy words and the reference words with explicit semantics. To conduct the empirical analysis with our framework, we used the dataset from the central bank in the united states (FRB), where the federal law requires them to publish the policy documents regularly to keep their policy's transparency, and their policy behavior is observable qualitative by their policy rate (the federal funds rate). Our simulation results with the estimated time series model well used for finance (VAR model) revealed that the policy authority (FRB) uses different documents to explain their policy changes depending on the phase of their policy. We also conducted the prediction analysis of the policy behavior to study the performance of the different time series models and language models. Even for the prediction, our proposed framework showed a better performance than the others. Those results may suggest that the proposed framework provides not only interpretable results but also a better predictor.

While we found the policy explanation's semantics dynamics, some issues remain that further research needs to address. First, we need to expand our dataset and prove that our results are consistent. For example, we could conduct the same analysis for other central banks such as the Bank of England, European Central Bank, or the Bank of Japan. Such a comparative study is feasible because our embedding method do not depend on a specific language. Moreover, our idea does not depend on specific word embedding model. Our paper's central argument is that we should not rely on the language features that do not directly capture the policy explanation. Therefore, if there is a method that captures the semantic shift of a given set of words in more sophisticated or robust way, we could apply our idea to that method. 


\section{References}

Federal Reserve Bank. 2020a. Board of governors of the federal reserve system, note $=$ https: / / www. federalreserve.gov/ monetarypolicy/fomc_historical.htm, last accessed May 10, 2020.

Federal Reserve Bank. 2020b. Fomc minute. https://stanford.edu/ rezab/ useful/fomc_minutes.html, last accessed May 10, 2020.

Federal Reserve Bank. 2020c. What economic goals does the federal reserve seek to achieve through its monetary policy? Https://www.federalreserve.gov/faqs/whateconomic-goals-does-federal-reserve-seek-toachieve-through-monetary-policy.htm.

Alan S Blinder, Michael Ehrmann, Marcel Fratzscher, Jakob De Haan, and David-Jan Jansen. 2008. Central bank communication and monetary policy: A survey of theory and evidence. Journal of Economic Literature, 46(4):910-45.

Valerio Di Carlo, Federico Bianchi, and Matteo Palmonari. 2019. Training temporal word embeddings with a compass. In Proceedings of the AAAI Conference on Artificial Intelligence, volume 33, pages 6326-6334.

Nikhil Garg, Londa Schiebinger, Dan Jurafsky, and James Zou. 2018. Word embeddings quantify 100 years of gender and ethnic stereotypes. Proceedings of the National Academy of Sciences, 115(16):E3635-E3644.

Petra M Geraats. 2002. Central bank transparency. The Economic Journal, 112(483):F532-F565.

William L Hamilton, Jure Leskovec, and Dan Jurafsky. 2016. Diachronic word embeddings reveal statistical laws of semantic change. arXiv preprint arXiv:1605.09096.

Spyros Makridakis, Evangelos Spiliotis, and Vassilios Assimakopoulos. 2018. Statistical and machine learning forecasting methods: Concerns and ways forward. PloS one, 13(3): 0194889.

James H Stock and Mark W Watson. 2001. Vector autoregressions. Journal of Economic Perspectives, 15(4):101-115.

James H Stock, Mark W Watson, et al. 2003. Introduction to Econometrics, volume 104. Addison Wesley Boston.

the Federal Reserve Bank of Chicago. 2020. The federal reserve's dual mandate. https: //www. chicagofed.org/research/ dual-mandate/dual-mandate, last accessed May 10, 2020.
Jason Welker. 2020. The economics classroom. https: / / econclassroom. com/glossary, last accessed May 10, 2020.

Theresa Wilson. 2005. Subjectivity lexicon. https://mpqa.cs.pitt.edu/lexicons / subj_lexicon/, last accessed May 10, 2020.

Theresa Wilson, Janyce Wiebe, and Paul Hoffmann. 2005. Recognizing contextual polarity in phraselevel sentiment analysis. In Proceedings of human language technology conference and conference on empirical methods in natural language processing, pages 347-354.

Michael Woodford. 2005. Central bank communication and policy effectiveness. Technical report, National Bureau of Economic Research. 